\title{
Research of the combined extremum-seeking control system dynamics on mathematical and computer models
}

\author{
Dmitriy Alexandrovich Bushuev ${ }^{1, *}$, Vasiliy Grigorievich Rubanov ${ }^{1}$, and Dmitriy Vitalyevich Titov $^{2}$ \\ ${ }^{1}$ BSTU n.a. V.G. Shukhov, Department of Engineering Cybernetics, 308012, Belgorod, Kostyukova Str, 46, Russia \\ ${ }^{2}$ SWSU, Department of Computer Science, 305040, Kursk, 50 let Oktyabrya Str., 94, Russia
}

\begin{abstract}
The article introduces the process of studying the dynamics of the combined extremum-seeking control system, which is used for grinding-mixing unit automatic balancing. The structure of the proposed system is shown and the algorithm of its operation is described. On the basis of the harmonic balance and Goldfarb's methods, the values of quality factors of the extremum-seeking control system on the first cycle were obtained in the absence of static map drift and the stability of the system was established. The cosimulation model of the combined extremum-seeking control system implemented in the MATLAB / Simulink software is presented and described. MSC.Adams model of the grinding-mixing unit, which is a plant in the co-simulation model of the system, is presented. The results of co-simulation of the extremum-seeking control system dynamics are presented and analysed.
\end{abstract}

\section{Introduction}

Extremum-seeking control (ESC) systems are designed to ensure the minimum or maximum of the plant cost function in real time of its operation.

ESC systems, depending on the seeking algorithm, are divided into the following [1]:

- Systems using the definition of derivative of the cost function;

- Stepper systems that respond to the sign and the value of the discrete increment of the output signal;

- Systems using determination of the direction of motion to the extremum by the phase shift between the input perturbation signal and the output signal;

- System using storing of the extremum, reacting to the difference between the optimum reached at the previous instants of time and the current value of the output and its derivatives.

Extremum-seeking control has found wide application in various sectors: in the automotive industry (anti-lock braking systems [2-3], internal combustion engines [4], electromechanical valves [5], cruise control systems [6], in aircraft construction (formation flight systems [7-8]), in robotics (autonomous robotics and mobile robots [9-11], manipulator positioning systems [12]), in the optimal adjustment of PI controllers [13], neural network and fuzzy controllers [14-15], and etc. [16]. In the Belgorod State Technological University named after V.G. Shukhov the combined ESC system for automatic balancing of centrifugal grinding-mixing (GMU) units has been proposed [17]. This system automatically changes the counterweights position during the work of the unit, in order to establish and maintain a minimum of supporting bearings vibration.
To reduce the losses for "search and yaw" inherent in ESC, the program control channel is included in this system which, starting from the second loading cycle of the grinding material, realize the movement of the counterweights along the drift trajectory found on the first cycle. In [18], the process of obtaining an approximate mathematical model of GMU is described and the results of the combined ESC dynamic simulation in the MATLAB/Simulink software are shown. There is a linear drift model for the plant parameters, in accordance with alternately loading the maximum amount of material into each of the grinding chambers.

However, often in practice, a single or batch dosing mode is used. To evaluate the performance of the proposed combined ESC method for GMU automatic balancing in the above dosing modes it is a necessity to perform dynamics co-simulation of the mechanical virtual plant, designed in the MSC.Adams software, and the control system, implemented in MATLAB/Simulink. This makes it possible to approach to the conditions for the operation of the automatic GMU vibration suppression system in real modes, and also to take into account the possible errors in the mathematical model that arose as a result of approximation. To compare the results of computer simulation with analytical calculations, as well as to assess the stability of the extremum-seeking (ES) control loop, a system analysis using approximated mathematical model and the harmonic balance method is performed in this paper.

\subsection{System description}

Figure 1 shows the functional diagram of the proposed system.

\footnotetext{
* Corresponding author: dmbushuev@gmail.com
} 


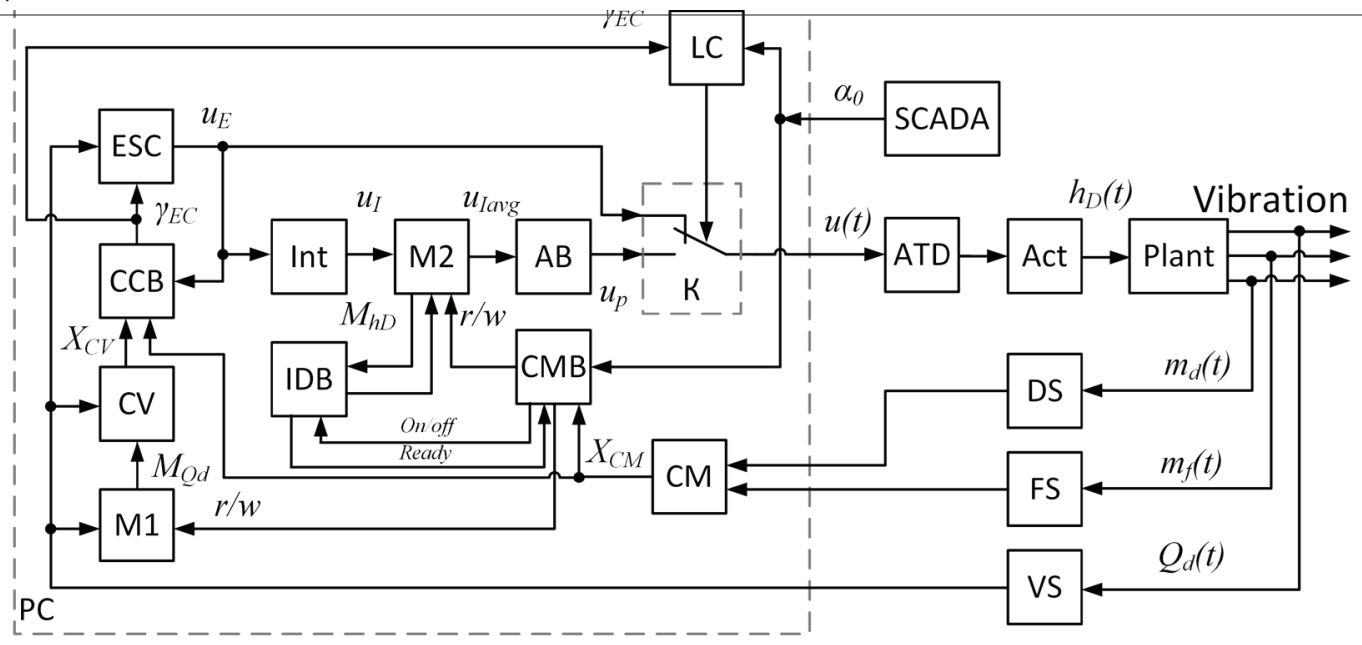

Fig. 1. Function block diagram of the system.

As an ESC algorithm in the ESC block uses a method of storing extremum with reverse zone defined by the necessary noise immunity. It is implemented movement of the counterweights with a constant speed by amplifying and transducing device ATD and actuator Act. The static map of the ES controller is shown in Figure 2.

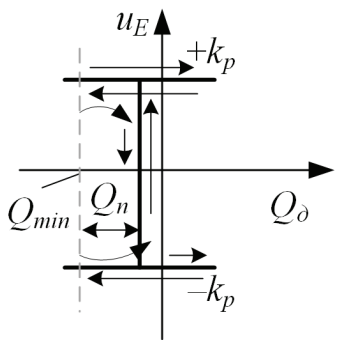

Fig. 2. The static map of the ES controller.

The combined control system, according to the algorithm in the programmable real-time controller PC, on the first cycle of material feeding which is determined by the appropriate loading signal $\alpha_{0}$ from the corresponding SCADA system, turn on the ES controller in the mode of constant extremum-seeking. At the same time, integrated using the integrator Int control signals from the ESC $U_{I}$ are stored in the memory block M2 and the corresponding values of the plant output $Q_{d}$ are recorded in the memory block M1. The termination of the grinding is determined in the mass comparator $\mathrm{CM}$ when the mass $m_{f}$ of the feed material is equal to the mass of discharged $m_{d}$, measured by the feed sensor FS and the discharge sensor DS, respectively. The $X_{C M}$ signal from the CM block via the memory control block CMB turns on drift identification block IDB that determines trajectory of drift by averaging (for example, using the moving average method with weighted local quadratic regression) and the resulting drift path is placed in the memory block M2 in a matrix form.

On subsequent grinding cycles, after the getting of the loading signal $\alpha_{0}$ and the measuring of the mass of the feed material, the memory control block CMB and the LC logic circuit (which is switching the key $\mathrm{K}$ ) sets the program control on the drift path obtained at the first cycle, converting it to the control action $u_{p}$ using the approximation block AB. In the case of an identical drift, program control system maintains the minimum of the plant output without seeking signals and yaw losses. At the same time, the current vibration level determined by the vibration sensor VS is constantly compared with the same value stored in memory M1. If the difference exceeds by the setting deadband, determined from the condition of the permissible deviation error, the vibration comparator $\mathrm{CV}$ sends $X_{C V}$ signal to the controller control block CCB, which starts the refinement seeking algorithm consisting in turning on the ESC in three movements with the last movement, equal to the halftime of the previous, thereby reducing the error of tracking the extremum accumulated as a result of program control. A more detailed algorithm of the system operation is described in [18].

\section{Research of the combined ESC system dynamics on the basis of a mathematical model}

Approximated dynamic mathematical model of threechamber grinding-mixing unit oscillations with an actuator in the form of differential equations is given by [17]:

$$
\left\{\begin{array}{l}
\frac{d}{d t}\left(T_{a} \frac{d h_{D}(t)}{d t}+h_{D}(t)\right)=k_{a} U(t), \\
Q\left(h_{D}, t\right)=\left\{\begin{array}{l}
k_{1}(t) \cdot\left(h_{D}-h_{D 0}(t)\right)^{2}+Q_{0}(t), h_{D} \leq h_{D 0}, \\
k_{2}(t) \cdot\left(h_{D}-h_{D 0}(t)\right)^{2}+Q_{0}(t), h_{D}>h_{D 0},
\end{array}\right. \\
T_{\text {out }} \frac{d Q_{d}}{d t}+Q_{d}=Q\left(t-\tau_{\text {Dout }}\right),
\end{array}\right.
$$

where $T_{a}$ - actuator time constant, $h_{D}$ - counterweights position, $k_{a}$ - transfer factor of the actuator, $U$-control action, $Q=f\left(h_{d}, t\right)$ - the static map of the plant, $k_{1}, k_{2}-$ the parabola coefficient, $h_{D 0}, Q_{0}$ - coordinate of the unimodal static map minimum, $Q_{d}$ - the output of the plant dynamic part, $T_{\text {out }}$ - the time constant of the plant dynamic part, $\tau_{\text {Dout }}-$ delay of the plant dynamic part. 
For the GMU virtual prototype, the numerical values of the quantities in the system of equations (1) are shown in Table 1.

Table 1. Quantities included in the plant description

\begin{tabular}{|c|c|}
\hline Parameter & Value \\
\hline$T_{a}, \mathrm{~s}$ & 0.1 \\
\hline$k_{a c t}, \mathrm{~cm} /(\mathrm{s} \cdot \mathrm{V})$ & 0.6 \\
\hline$k_{1}, \mu \mathrm{m} / \mathrm{cm}$ & 17.8 \\
\hline$k_{2}, \mu \mathrm{m} / \mathrm{cm}$ & 13.2 \\
\hline$h_{D 0}, \mathrm{~cm}$ & 9.418 \\
\hline$Q_{0}, \mu \mathrm{m}$ & 253.9 \\
\hline$T_{\text {out }}, \mathrm{s}$ & 0.01 \\
\hline$\tau_{\text {Dout }}, \mathrm{s}$ & 0.147 \\
\hline
\end{tabular}

On the first cycle of material feeding, the control action $\mathrm{u}(\mathrm{t})$ is formed only by the ES controller, which works in the seeking mode. For such a nonlinear system, the structure and filter hypothesis is fulfilled (since the actuator and the plant dynamic part exhibit the properties of a low-pass filter), then for the dynamics analysis it is possible to use the method of harmonic balance. Quality factors of the ESC system: the amplitude $a_{l}$ and $\omega$ is the frequency of self-oscillations in the absence of drift (i.e. $\left.Q_{0}=h_{D 0}=0\right)$ can be found by using the Goldfarb's method.

According to the Nyquist criterion, the appearance of a self-oscillation mode in a closed harmonic linearized system occurs when the following condition is satisfied

$$
W_{e}(j \omega)=-\frac{1}{J_{2}\left(a_{1}\right)},
$$

where $W_{e}(j \omega)$ is the equivalent complex frequency response of the plant with an actuator equal to

$$
W_{e}(j \omega)=\frac{2 k_{p}\left(k_{1}+k_{2}\right)}{\pi} \cdot\left(W_{a}\left(j \frac{\omega}{2}\right)\right)^{2} W_{D}(j \omega) e^{-j \frac{\pi}{2}},
$$

and $J 2\left(a_{1}\right)$ is the equivalent complex transfer factor of the nonlinear ES controller, which, as a result of the harmonic linearization of the static map (see Fig. 2), can be defined as

$$
J_{2}\left(a_{1}\right)=\frac{4 k_{p}}{\pi a_{1}} e^{j\left(\pi-\arcsin \frac{Q_{H}-a_{1}}{a_{1}}\right)},
$$

where $Q_{n}$ is the dead space of ES controller, accepted for this plant (equal to $10 \mu \mathrm{m}$ ).

To solve equation (2) with respect to $a_{1}$ and $\omega$, amplitude-phase characteristics of $W_{e}(j \omega)$ and $-1 / J 2\left(a_{1}\right)$ according to Goldfarb's method are constructed (Fig. 3). As a result, it was found that the amplitude of the oscillations $a_{1}=5.3 \mu \mathrm{m}$ and the frequency $\omega=1.84 \mathrm{rad} / \mathrm{s}$. The found periodic solution is stable, since with increasing amplitude $a_{1}$ from 0 to inf the curve -1/ J2 $\left(a_{1}\right)$ crosses the complex frequency characteristic of the linear part $W_{e}(j \omega)$ in the direction from the inside outward. In the case of the static map drift the differential equations (1) are non-autonomous, which makes it difficult to use analytical methods to estimate the dynamics of the ESC. Therefore, to analyze the dynamics of the ESC system with the drift of a static map, computer simulation is used.

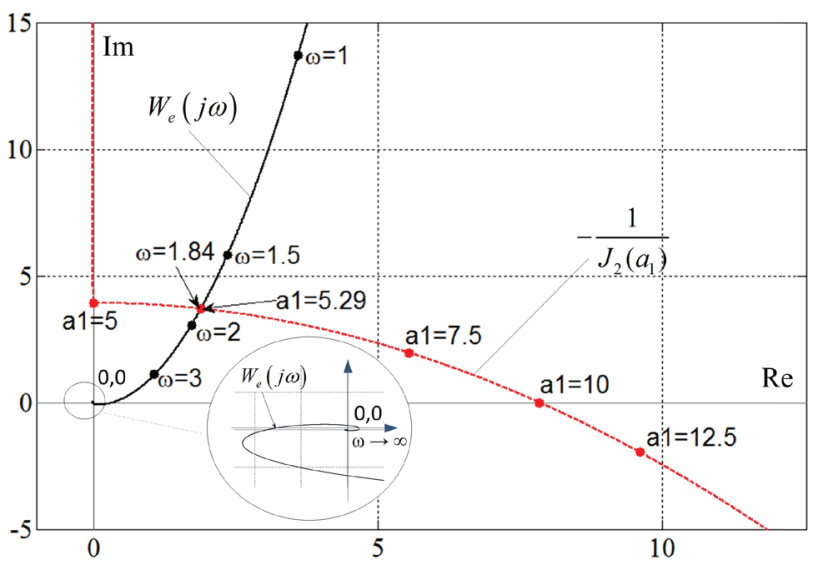

Fig. 3. Determination of the amplitude and frequency of selfoscillations of the ESC by the Goldfarb's method

\section{Co-simulation model of the combined ESC system}

A dynamic model of the combined ESC system with the integration of a virtual prototype of GMU with automatic balancing created in the MSC.Adams software is shown in Fig. 4.

The functions of the memory block M2 and drift identification block IDB are performed by the "Avg" subroutine, which performs the averaging using weighted local quadratic regression. The "PSGoal" block, in accordance with the drift trajectory obtained on the first cycle, sets the value of the speed that is fed to the integrator "Integrator" of the actuator. The ES controller is executed using two subprograms in the form of S-functions: "ESCLC" defines a learning search by the method of storing an extremum on the first feeding cycle, "ESCWC" performs the functions of the ES controller on the remaining cycles and the M1 memory block in the training mode. To control of the different modes turning on, the sub-programs Subsystem1 and Subsystem are used. Relay element K is implemented using the "Relay" block.

To create a kinematic connection between the eccentric and additional shaft, a gear module from Adams/Machinery is used and a "Bearing" is used in the "Compliant" mode to set the bearings, which allows approximately obtaining the oscillations of the GMU bearing supports. The radial stiffness of the bearings is set equal to $k=1.23 \times 10^{4} \mathrm{~N} / \mathrm{cm}$, and the damping factor $c=10 \mathrm{~N} \cdot \mathrm{s} / \mathrm{cm}$. In order to change the grinding material loading $\triangle m$, during the GMU operation, GFORCE forces are added to each CM_marker of the grinding chambers. The format of such functions is $\mathrm{FX}=\operatorname{Varval}($ deltam)* *ACCX(CM marker), where ACCX is the function that determines the acceleration along the $\mathrm{X}$-axis, for 


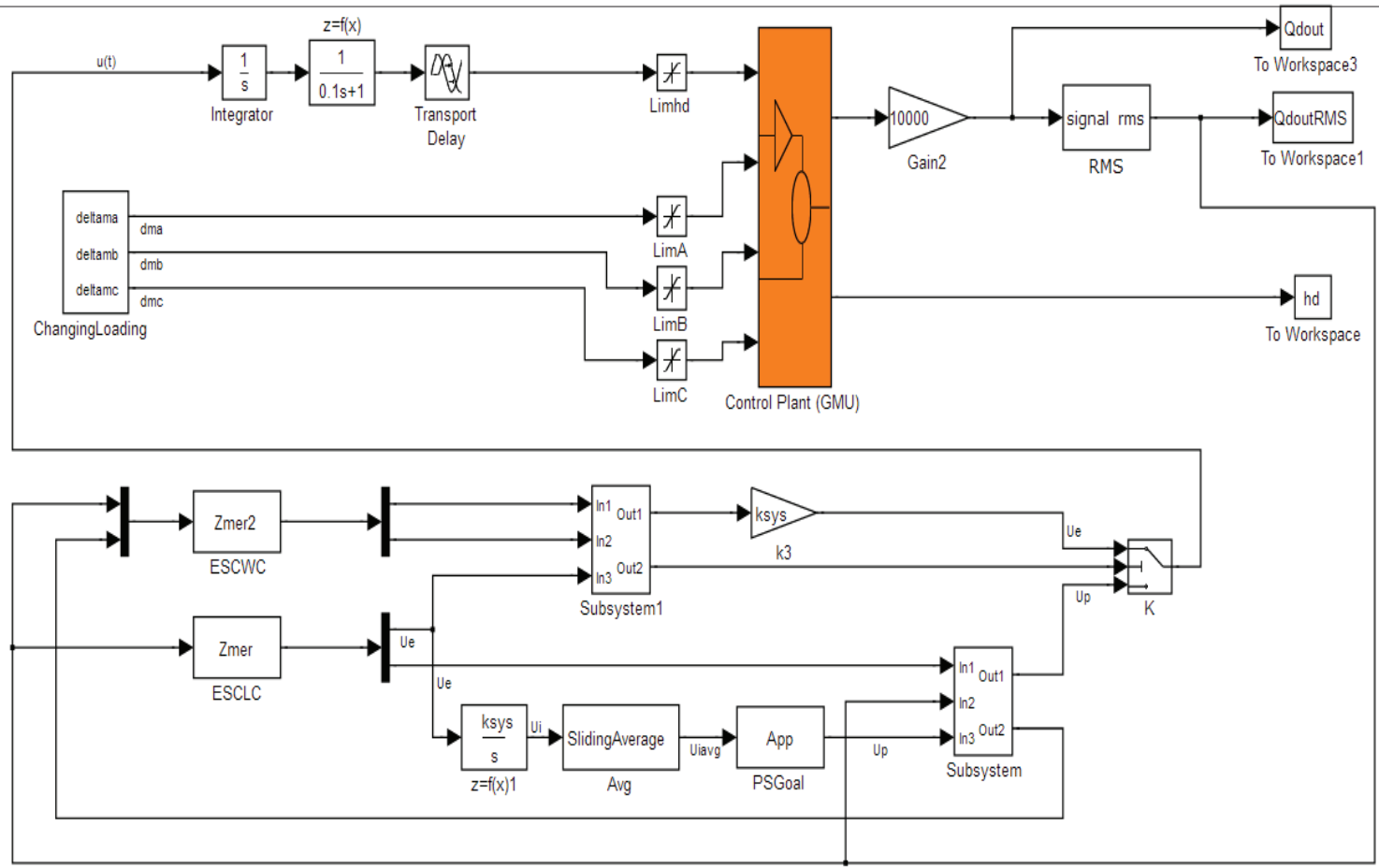

Fig. 4. Co-simulation model of the combined ESC system. example, to change the loading of the grinding chamber A, the function FX takes the following form: Varval(deltama)*ACCX(A.cm)-Varval(deltama)*g.

The model of the GMU in the MSC.Adams software is presented in Fig. 5.

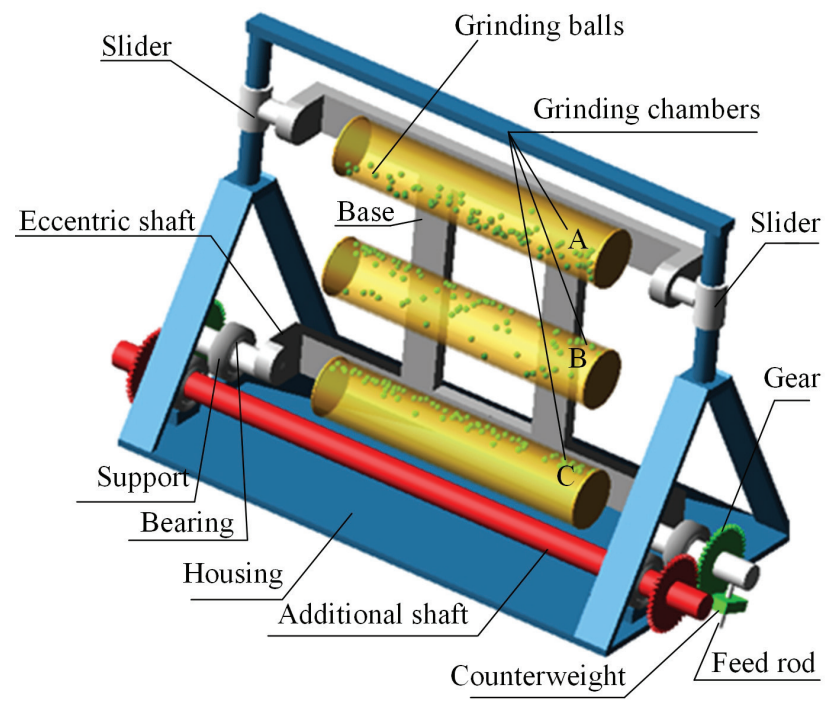

Fig. 5. Adams model of the GMU.

\section{Simulation results and discussion}

In the virtual GMU prototype, the load factor of grinding bodies in the grinding chambers is determined by the $K_{L}=25 \%$, which corresponds to a mass of $13.8 \mathrm{~kg}$. Two variants of the periodic operation of the unit with automatic balancing were simulated:

- Single dosing of the maximum amount of grinding material into the upper grinding chamber;

- Batch dosing of grinding material into the upper grinding chamber.
For the first variant, the "ChangeLoading" subroutine specifies the change in the loading of grinding chambers, shown in Fig.6. In this case, the maximum amount of material in the lower chamber is not more than $9 \mathrm{~kg}$.

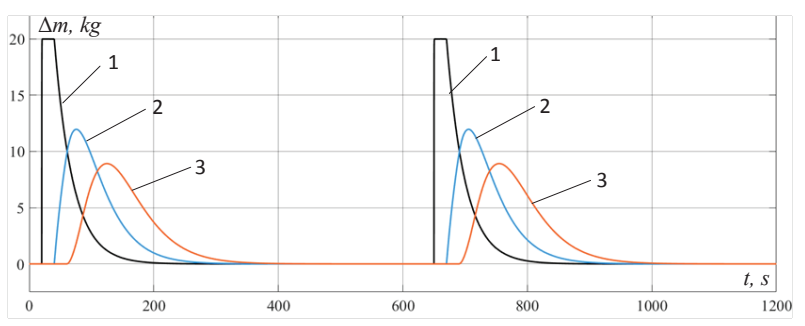

Fig. 6. Graphs of changing the loading of the GMU grinding chambers with a single dosing of the maximum amount of grinding material: 1 - chamber $\mathrm{A}\left(\Delta m_{A}\right), 2$ - chamber $\mathrm{B}\left(\Delta m_{\mathrm{B}}\right)$, 3 - chamber $\mathrm{C}\left(\Delta m_{C}\right)$.

As a result of the combined ESC system dynamic simulation, the following results were obtained (Figures 7-8).

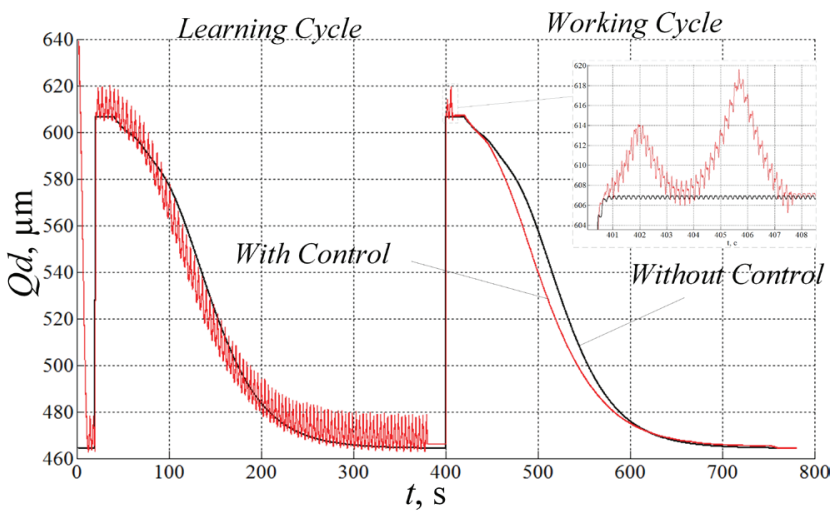

Fig. 7. RMS of the virtual prototype vibration displacement in a single dosing mode. 


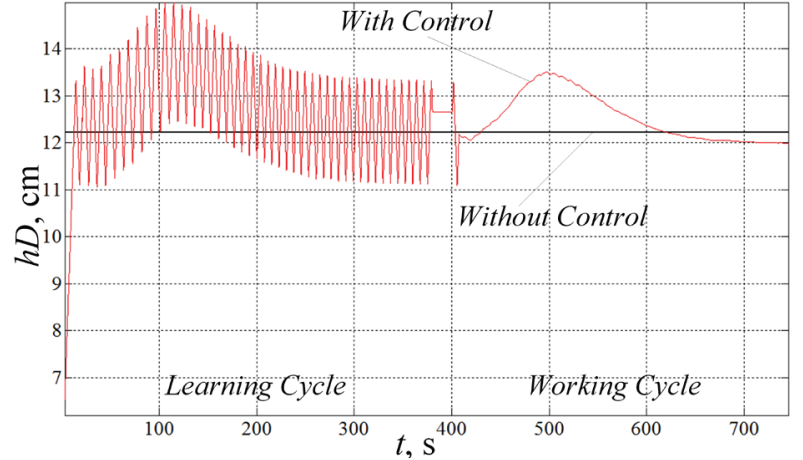

Fig. 8. Counterweights position.

At the initial moment of the GMU operation, the position of the counterweights is $h_{D}=6.5 \mathrm{~cm}$ and the RMS of the vibration displacement $Q_{D}=640 \mu \mathrm{m}$. The work of the ES controller on the first cycle of operation allowed to search for the minimum value of vibration $(464 \mu \mathrm{m})$, thereby reducing the level of oscillations by $28 \%$, after which a yaw is observed near this value. As can be seen, the values of the amplitude and frequency of the output plant oscillations in the absence of the drift coincide with the results obtained from the analysis by the harmonic balance method of the system with the plant given by the mathematical model. On the second grinding cycle, the ES controller was turned on once when the set tracking error threshold was exceeded at the beginning after the material was loaded, thus, yawing was practically absent. The maximum decrease in the level of RMS vibration displacement compared to the level corresponding to the fixed position of the counterweights $\left(h_{D}=12.2 \mathrm{~cm}\right)$ on the training cycle is $20.5 \mu \mathrm{m}(3.7 \%)$ and in the mode of the program control $18.5 \mu \mathrm{m}(3.4 \%)$.

For a comparative evaluation of the vibration suppression efficiency, the integral index $I_{e f}$, is represented in the form

$$
I_{e f}=\frac{1}{t_{2}-t_{1}} \int_{t_{1}}^{t_{2}}\left(Q_{w c}(t)-Q_{c}(t)\right) d t
$$

where $t_{1}, t_{2}$ - initial and final moments of the efficiency calculation, $Q_{w c}(t), Q_{c}(t)$ are the values of the control plant output without a control and with a regulator, respectively.

The value of the integral index in the interval from $t_{1}=20 \mathrm{~s}$ to $t_{2}=780 \mathrm{~s}$ (i.e., from the beginning of the grinding process to the end of the second cycle) is equal to $I_{e f}=1.65$.

In the batch mode, the operation of the combined system was investigated by grinding $42 \mathrm{~kg}$ of material, dosed at $14 \mathrm{~kg}$ per portion, dispensed at a given time interval.

The "ChangeLoading" subroutine specifies the following change in grinding load (Fig. 9). The maximum amount of material in the bottom grinding chamber is not more than $12.7 \mathrm{~kg}$.

The co-simulation results of the combined ESC system of the GMU automatic balancing with the load factor of grinding bodies $K_{L}=25 \%$ are shown in Fig. 10 .

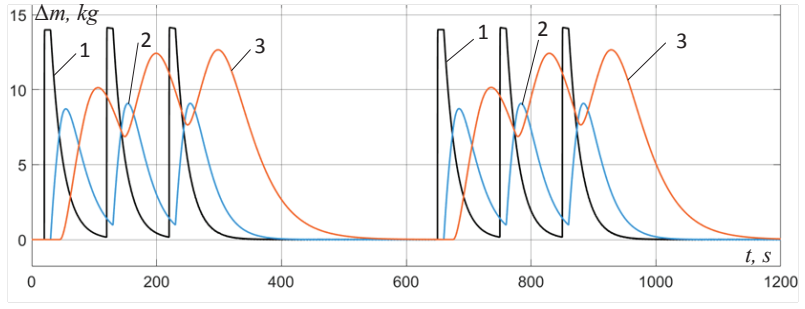

Fig. 9. Graphs of changing the loading of the GMU grinding chambers with a batch dosing of the maximum amount of grinding material: 1 - chamber $\mathrm{A}\left(\Delta m_{A}\right), 2$ - chamber $\mathrm{B}\left(\Delta m_{\mathrm{B}}\right)$, 3 - chamber $\mathrm{C}\left(\Delta m_{C}\right)$.

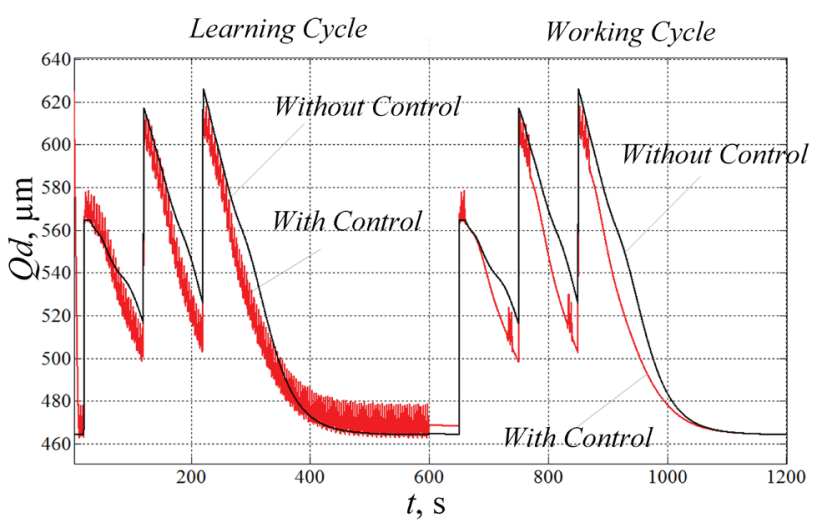

Fig. 10. RMS of the virtual prototype vibration displacement in a batch dosing mode.

Using of the combined ESC system on the training cycle ensured a maximum reduction in the level of vibration displacement RMS by $31 \mu \mathrm{m}(5.6 \%)$, but there were large yaw losses. In a while program control ensured yawing only at the moments corresponding to a strong drift of the static map, and this was also facilitated by the low value of the deadband $(5 \mu \mathrm{m})$, above which the ES controller was turned on for three seeking oscillations. The maximum decrease in the level of oscillations in the working cycle was $30 \mu \mathrm{m}(5.5 \%)$. The integral efficiency index over the interval from $t_{1}=20 \mathrm{~s}$ to $t_{2}=1200 \mathrm{~s}$ (on two grinding cycles) exceeded the value of $E_{\text {ef }}=7.42$.

Increasing the load factor of grinding bodies $K_{L}$ to a level of $30 \%(16.6 \mathrm{~kg})$ leads to a shift of the extremum position, as a result of which the balance error with the aid of a fixed located counterweights increases (Fig. 11).

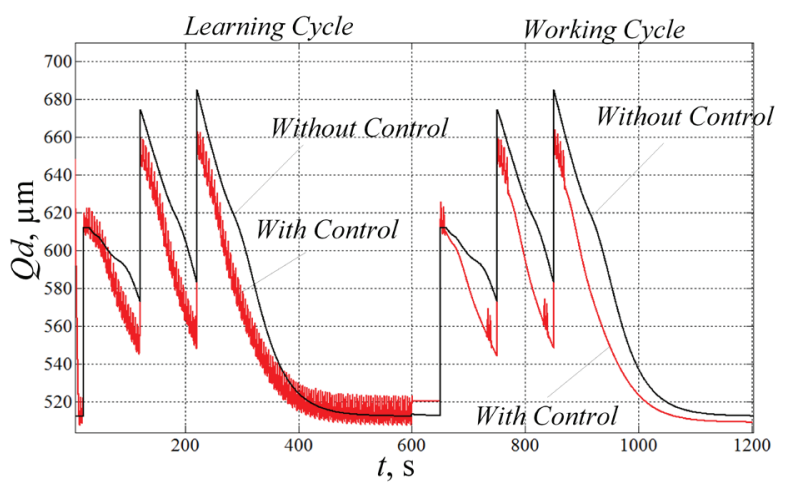

Fig. 11. RMS of the virtual prototype vibration displacement in a batch dosing mode with $K_{L}=30 \%$. 
Using the developed system, it was possible to significantly reduce the average level of oscillations (the efficiency index $I_{e f}$ is more than 15.1 in the interval from $t_{1}=20 \mathrm{~s}$ to $t_{2}=1200 \mathrm{c}$.) The maximum decrease in the vibration displacement RMS level was $44 \mu \mathrm{m}$ (7.5\%).

For the comparative efficiency evaluation of the combined ESC system on the training (when combined ESC system operated as a classical ESC system with an extremum storing) and the working cycles, in comparison with the fixed counterweight, indexes $I_{e f}$ were also determined at the corresponding time intervals. The results of a comparative analysis of the combined ESC system efficiency are presented in Table 2.

Table 2. Comparative analysis of the combined ESC system efficiency

\begin{tabular}{|c|c|c|c|c|}
\hline & \multicolumn{3}{|c|}{ Efficiency index, $\boldsymbol{I}_{\text {ef }}$} \\
\cline { 2 - 5 } $\begin{array}{c}\text { Dosing } \\
\text { mode }\end{array}$ & $\begin{array}{c}\text { Training } \\
\text { Cycle }\end{array}$ & $\begin{array}{c}\text { Working } \\
\text { Cycle }\end{array}$ & $\begin{array}{c}\text { The } \\
\text { Both } \\
\text { Cycles } \\
\text { in } \\
\text { efficiency } \\
\text { due to the } \\
\text { program } \\
\text { control }\end{array}$ \\
\hline $\begin{array}{c}\text { Single } \\
\left(\begin{array}{c}20 \mathrm{~kg}, \\
K_{L}=25 \\
\%)\end{array}\right.\end{array}$ & -0.3 & 3.6 & 1.65 & $\begin{array}{c}\text { more than } \\
100 \%\end{array}$ \\
\hline $\begin{array}{c}\text { Batch } \\
(42 \mathrm{~kg}, \\
K_{L}=25 \\
\%)\end{array}$ & 5.6 & 9.2 & 7.42 & $39 \%$ \\
\hline $\begin{array}{c}\mathrm{Batch} \\
(42 \mathrm{~kg}, \\
K_{L}=30 \\
\%)\end{array}$ & 13.7 & 16.53 & 15.1 & $17 \%$ \\
\hline$\%$
\end{tabular}

\section{Conclusions}

The application of the program control algorithm in the combined system is much more effective than using an ESC system with an extremum storing due to the presence of search and yaw losses in the last one. Based on the results of co-simulation, it is also evident that the effectiveness of reducing the level of undesired oscillations increases with increasing degree of filling of the bottom grinding chamber with a material or grinding bodies.

Using a continuous loading mode with a constant feed rate for grinding a limited amount of material (for example, the same $42 \mathrm{~kg}$ ) can cause a significant reduction in the accuracy of balancing by the fixed counterweights at the start and in the end of grinding process, when not all the chambers are full. Therefore, using of the developed system is also justified in the continuous loading mode. The proposed control principle can be applied to the extremum-seeking control of low-inertia plants with unknown unimodal static maps with cyclic drift.
Research is carried out with the financial support of The Ministry of Education and Science of the Russian Federation within the Public contract project 2.1396.2017/PCh.

\section{References}

1. E.S. Rybchikova, M.Yu. Rybchikov, A.I. Sunargulova, R.V. Tankov, V.Yu Perevalov, Mechatronics., Automation., Control, 16, 5 (2015)

2. S. Drakunov, "U. "Ozg"uner, P. Dix, B. Ashra, IEEE Trans. on Control Sys. Tech., 3, pp. 79-85 (1995)

3. C. Zhang and R. Ord'or nnez, IEEE Trans. on Autom. Control, 52, 3 (2007)

4. N. J. Killingsworth, S. M. Aceves, D. L. Flowers, F. Espinosa-Loza, M. Krstic, IEEE Trans. on Control Sys. Tech., 17, 6 (2009)

5. K. S. Peterson, A. G. Stefanopoulou, Automatica, 40, pp. 1063-1069 (2004)

6. A. Rahnama, M. Xia, S. Wang, P.J Antsaklis, Proc. of the American Control Conf., art. no. 7526629, pp. 6109-6114 (2016)

7. P. Binetti, K.B. Ariyur, M. Krstić, F. Bernelli Proc. of the American Control Conf., 4, pp. 2848-2853 (2002)

8. B. Zuo, Y. A. Hu, Proceedings of Fifth World Congress on Intelligent Control and Automation, pp. 3302-3305, (2004)

9. J. Cochran, M. Krstic, IEEE Trans. on Automatic Control, 54, 4, (2009)

10. C. G. Mayhew, R. G. Sanfelice, A. R. Teel, Proc. of the 2007 American control conf., pp. 1185-1190 (2007)

11. M. S. Stankovic, D. M. Stlipanovic, Proc. of the 48th IEEE Conf. on Decision and Control, pp. 4541-4546 (2009)

12. V. Koropouli, A. Gusrialdi, S. Hirche, D. Lee, Control Engineering Practice, 52, pp. 1-14 (2016)

13. M. Guay, D.A. Dochain, Automatica, 77, pp. 61-67 (2017)

14. L. Gurvich Proc. of the IEEE Convention of Electrical and Electronics Engineers, pp. 18-21 (2004)

15. Y. A. Hu, B. Zuo, Int. J. of Inf. Tech., 11, 6, (2005)

16. Y. Tan, W.H. Moase, C. Manzie, D. Nesic, I.M.Y. Mareels, Proc. of 29th Chinese control conf., pp 1426 (2010)

17. D.A. Bushuev, V.G Rubanov, D.V. Velichko, ARPN Journal of Engineering and Applied Sciences, 11, pp. 12288-12294 (2016)

18. V.G. Rubanov, D.A. Bushuev Patent $R U$ No. 2600688 (2016) 